\title{
REVISÃo de LITERATURA Influência familiar na anorexia nervosa: em busca das melhores evidências científicas
}

\author{
Family influence on anorexia nervosa: in search of the best scientific evidence
}

Élide Dezoti Valdanha', Fabio Scorsolini-Comin², Rodrigo Sanches Peres ${ }^{3}$, Manoel Antônio dos Santos ${ }^{4}$

\section{RESUMO}

Objetivo: Analisar as evidências científicas sobre a influência do grupo familiar na sintomatologia da anorexia nervosa (AN). Métodos: Revisão sistemática da literatura no período de 2000 a 2012, utilizando as bases PubMed, CINAHL, PsycINFO, Lilacs e os descritores: "transtornos da alimentação", "relações familiares", "relação entre gerações" e "relação mãe-filho". Resultados: Com base nos critérios de inclusão e exclusão, foram selecionadas e analisadas 20 publicações na íntegra. A maior parte dessas publicações focaliza mulheres diagnosticadas com transtornos alimentares (TA) ou com comportamento alimentar de risco, tem delineamento quantitativo e utiliza escalas padronizadas para coleta de dados. Predominou o Nível de Evidência 4, que corresponde a estudos de desenho não experimental, como pesquisa descritiva correlacional e qualitativa, ou estudos de caso. As evidências encontradas indicam que os relacionamentos familiares exercem impacto significativo tanto no desenvolvimento como na manutenção de sintomas de TA. Padrões relacionados à alimentação e ao alimento são transmitidos entre as gerações, bem como aspectos não elaborados do funcionamento psíquico materno. Conclusões: Os estudos sugerem a necessidade de ampliar o foco da atenção para incluir os familiares no tratamento, bem como incorporar os achados de transmissão psíquica intergeracional como subsídios norteadores do planejamento e qualificação do cuidado oferecido nos TA.

\section{ABSTRACT}

Objective: To analyze scientific evidence about the influence of the family group on anorexia nervosa (AN) symptoms. Methods: Systematic review of the literature published between 2000 and 2012, using the databases PubMed, CINAHL, PsycINFO, Lilacs and the descriptors: "eating disorders", "family relations", "intergenerational relations" and "mother-child relations". Results: Based on the inclusion and exclusion criteria, 20 publications were selected and their full version was analyzed. Most of the papers focus on women diagnosed with eating disorders (ED) or risky eating behavior, have a quantitative design and use standardized scales for data collection. Evidence Level 4 was predominant, which corresponds to studies with a non-experimental design, like correlational and qualitative descriptive studies, or case stu-

1 Universidade de São Paulo, Faculdade de Filosofia, Ciências e Letras de Ribeirão Preto (USP/FFCLRP), Programa de Pós-Graduação em Psicologia; USP/FFCLRP, Laboratório de Ensino e Pesquisa em Psicologia da Saúde (LEPPS). Faculdade de Medicina de Ribeirão Preto da Universidade de São Paulo, Hospital das Clínicas, Grupo de Assistência em Transtornos Alimentares (FMRP-USP-HC/GRATA). 


\section{Keywords}

Eating disorders, family relations, intergenerational relations, mother-child relations. dies. The evidences found indicate that family relations significantly affect both the development and maintenance of ED symptoms. Eating and food patterns are transmitted between generations, as well as non-elaborated aspects of maternal psychic functioning. Conclusions: Studies suggest the need for a broader care focus, so as to include family members in treatment, as well as to incorporate the findings related to intergenerational psychic transmission to guide the planning and qualification of the care offered in case of ED.

\section{INTRODUÇÃO}

A prática baseada em evidências (PBE) é um paradigma emergente, utilizado principalmente em situações que geram incertezas quanto aos aspectos de diagnóstico, prognóstico e manejo terapêutico'. A PBE apoia-se nos mesmos conceitos da Medicina baseada em evidências, com a diferença de que é empregada por diferentes profissionais e em contextos de saúde mais variados. Esse paradigma exige que o profissional desenvolva competência em avaliar e validar criticamente as publicações científicas em termos do nível de evidência (NE).

O NE de um estudo é uma classificação utilizada para ranquear a validade do diagnóstico, a conduta terapêutica e o prognóstico, e qualificar a informação descrita nas publicações científicas². No contexto da saúde, a preocupação com o NE provém da PBE, interessada em classificar os estudos pautados em estratégias metodológicas rigorosas, que possam dar respaldo à prática profissional. A efetividade e a eficiência nas condutas exigem a integração da experiência do profissional com as melhores evidências extraídas, de preferência, das revisões sistemáticas ${ }^{3}$.

Para avaliar as evidências disponíveis, o profissional/ pesquisador de saúde deve compreender e analisar a metodologia científica que embasa o estudo33. De acordo com a classificação da Agency for Healthcare Research and Quality (AHRQ), as evidências são categorizadas em seis níveis. O NE 1 corresponde à metanálise de múltiplos estudos controlados, ao passo que o NE 2 remete a estudos individuais com delineamento experimental. O NE 3 abrange pesquisas com desenho quase-experimental, como estudos sem randomização com grupo único pré e pós-teste, longitudinais ou caso-controle, e o NE 4 se refere a estudos não experimentais, como pesquisas descritivas correlacionais e qualitativas ou estudos de caso. Relatórios de casos ou avaliações de programas apresentam NE 5 e, por fim, o NE 6 se aplica a opiniões de autoridades respeitadas na área ou de especialistas, incluindo informações não fundamentadas em pesquisas ${ }^{4}$.

O presente estudo visa familiarizar o profissional de saúde mental com os diferentes NE disponíveis no campo dos transtornos alimentares (TA), para que a tomada de decisão na prática assistencial seja fundamentada nas melhores evi- dências disponíveis. Os TA constituem quadros psiquiátricos de etiopatogenia multifatorial. Os tipos mais relevantes anorexia nervosa (AN) e bulimia nervosa (BN) - têm como fatores desencadeadores e mantenedores: aspectos biológicos e genéticos, meio sociocultural, funcionamento familiar e personalidade do indivíduo ${ }^{5,6}$.

No que se refere às relações familiares, a associação entre AN e processos psíquicos pode ser constatada em diferentes gerações de uma mesma família?. Essa consideração evoca os postulados teóricos acerca da transmissão psíquica entre gerações, que entendem a família como um espaço psíquico comum, que possibilita os processos de construção, organização e transformação dos vínculos e dos conteúdos emocionais que são transmitidos de uma geração a outra-11.

Na construção de uma nova família, ocorre o cruzamento das genealogias paterna e materna, que colocam em marcha tanto identificações como contraidentificações. Esse processo mobiliza forças intensas de amor e ódio, que se aglutinam em torno do que é aceito e também do que é denegado e escamoteado da consciência, não sendo possível controlar aquilo que se transmite ${ }^{12}$. Desse modo, tanto os aspectos adaptativos (vinculados à sobrevivência) como os disfuncionais (relacionados ao desencadeamento de psicopatologias, por exemplo) podem ser transmitidos, em nível inconsciente, de uma geração a outra do grupo familiar ${ }^{13}$.

A pergunta de pesquisa que norteou o presente estudo foi: existem evidências científicas, na literatura indexada, que corroboram as hipóteses acerca do nexo entre AN e processos psíquicos transmitidos entre as gerações de uma mesma família? Em caso afirmativo, como os padrões intergeracionais repercutem na sintomatologia?

O presente estudo focalizou a produção científica consagrada às relações familiares de pacientes com AN, considerando que esse tema vem adquirindo proeminência no cenário científico em virtude da valorização da dinâmica familiar disfuncional como fator crucial na compreensão etiológica do problema ${ }^{5,6,14}$. Assim, tem sido apontada a necessidade de intervenções que incluam a família e a questão da saúde mental do grupo familiar ${ }^{15}$. Porém, pouco se conhece sobre o status da evidência científica dos estudos que, dentro do campo mais abrangente das relações familiares, se dedicam a investigar os processos de transmissão psí- 
quica intergeracional na AN. Partindo dessas considerações, o presente estudo teve por objetivo analisar as evidências científicas disponíveis sobre a influência do grupo familiar na sintomatologia da AN.

\section{MÉTODOS}

O presente estudo configura uma revisão sistemática da literatura. Para a busca dos artigos que constituíram o corpus que deu suporte a essa revisão, foram selecionadas quatro bases de dados: PubMed, CINAHL, PsycINFO e Lilacs. Foram utilizadas as seguintes palavras-chave, extraídas dos Descritores em Ciências da Saúde (DeCS) e combinadas entre si: "transtornos da alimentação", "anorexia nervosa", "relações familiares", "relação entre gerações" e "relação mãe-filho". O termo "transmissão psíquica" não foi utilizado por não estar indexado no DeCS.

Foram incluídos artigos que preenchiam os seguintes critérios: resumo disponível nas bases de dados consultadas; publicação em língua portuguesa, inglesa ou espanhola; período de publicação de janeiro de 2000 a dezembro de 2012; com temáticas pertinentes à questão norteadora desta revisão. Foram incluídos artigos com vários tipos de delineamento, de modo a abarcar a abrangência do conhecimento produzido acerca da temática escolhida. Foram excluídos livros, capítulos de livro, resenhas, cartas, notícias, dissertações e teses, além de artigos de revisão da literatura e estudos com temática distante das relações familiares de pacientes com AN.

A busca nas bases de dados foi seccionada em quatro etapas. Na primeira, foi realizada uma busca geral nas bases de dados selecionadas, com as combinações dos descritores previamente escolhidos. Na segunda etapa, os resumos foram lidos e analisados segundo os critérios de inclusão e exclusão adotados. Já na terceira etapa foram excluídos os resumos repetidos. Na quarta e última etapa foi realizada a leitura dos artigos resultantes da terceira etapa, de modo que apenas aqueles que estavam diretamente relacionados à temática desta revisão foram selecionados. Estes foram lidos na íntegra e analisados em profundidade por dois avaliadores. Na apreciação do material obtido buscou-se evidenciar o número e o perfil dos estudos disponíveis, bem como avaliar suas características metodológicas, classificando-os em função do delineamento de pesquisa e NE científica de acordo com a categorização da $\mathrm{AHRQ}^{4}$.

\section{RESULTADOS}

A quantidade de artigos localizados, excluídos, incluídos e selecionados com base nas quatro etapas delineadas neste estudo pode ser visualizada na figura 1. A maioria dos artigos foi recuperada na base de dados PubMed ( $n=11)$. Na base CINAHL foram recuperados seis artigos, na PsycINFO dois e na Lilacs apenas um. Assim, foram analisados na íntegra 20 artigos, os quais constituíram o corpus que deu suporte à presente revisão. A expressiva redução do número de estudos localizados para os efetivamente recuperados deu-se em função dos critérios de inclusão e exclusão adotados. A maior parte dos estudos foi excluída por tratar de modo tangencial a influência da família na sintomatologia da AN ou por apenas mencionar o grupo familiar como importante nos estudos sobre a AN, sem abordá-lo diretamente.

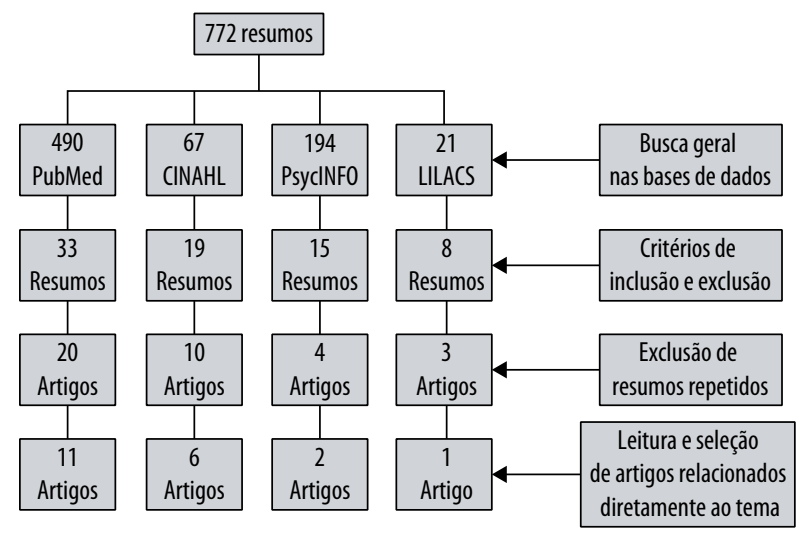

Figura 1. Fluxograma dos resultados da revisão sistemática.

Nos artigos revisados predominou o NE 4 (13 artigos), que corresponde a estudos de desenho não experimental, como pesquisa descritiva correlacional e qualitativa, ou estudos de caso. Também foram encontrados sete artigos com NE 3, que abrange pesquisas com desenho quase-experimental, pré e pós-teste, ou grupo controle, ou recorte longitudinal. Nas tabelas 1 e 2 é possível examinar autoria, ano de publicação, país de origem, participantes, delineamento do estudo e principais resultados dos artigos com NE 3 e 4, respectivamente. Não foram encontrados artigos pertinentes à temática aqui analisada com NE 1 ou 2, que corresponderiam a delineamentos robustos, como estudos de metanálise ou experimentais controlados ${ }^{3,4}$.

De modo geral, as populações investigadas nos estudos abrangem mulheres com TA, principalmente AN, sobretudo adolescentes do sexo feminino e mulheres jovens, que já apresentaram o quadro clínico ou não. Além disso, também foram incluídos nos estudos familiares de pacientes com TA ou, mais especificamente, mães de crianças ou de jovens com TA, bem como constituindo grupos controle, mulheres sem TA ou familiares de pessoas não diagnosticadas com TA. Os artigos focalizam os vínculos e conflitos familiares e suas possíveis influências tanto no desenvolvimento como na manutenção dos TA; padrões de comportamento e hábitos alimentares presentes no grupo familiar; e conteúdos intergeracionais, ou seja, transmitidos a cada geração, relacionados aos TA. 
Tabela 1. Caracterização dos artigos com NE 3 segundo primeiro autor/ano de publicação, origem, características dos participantes, delineamento metodológico do estudo e principais resultados

\begin{tabular}{|c|c|c|c|c|}
\hline Autoria/Ano & Origem & Participantes & Delineamento do estudo & Principais resultados \\
\hline $\begin{array}{l}\text { Eisenberg et al. } \\
\text { (2012) }\end{array}$ & Estados Unidos & 1.902 jovens & $\begin{array}{l}\text { Quase-experimental } \\
\text { (longitudinal - } 10 \text { anos) }\end{array}$ & $\begin{array}{l}\text { Mulheres que recebem com frequência comentários negativos de pessoas } \\
\text { importantes em sua vida têm maior probabilidade de desenvolver sintomas } \\
\text { na esfera do comportamento alimentar }\end{array}$ \\
\hline Micali et al. (2011) & Reino Unido & $\begin{array}{l}441 \text { mulheres diagnosticadas com } \\
\text { TA, } 10.461 \text { mulheres sem diagnóstico } \\
\text { de transtorno psiquiátrico e os filhos } \\
\text { dessas mulheres }\end{array}$ & $\begin{array}{l}\text { Quase-experimental } \\
\text { (longitudinal - } 1 \text { ano) }\end{array}$ & $\begin{array}{l}\text { Observou-se que sintomas de TA ativos no período de gravidez estão } \\
\text { associados a dificuldades alimentares do bebê, que ocasionam baixo peso, } \\
\text { além de outros prejuízos desenvolvimentais }\end{array}$ \\
\hline Field et al. (2008) & Estados Unidos & 12.534 adolescentes de ambos os sexos & $\begin{array}{l}\text { Quase-experimental } \\
\text { (longitudinal - } 7 \text { anos) }\end{array}$ & $\begin{array}{l}\text { Cerca de } 10 \% \text { das meninas e } 3 \% \text { dos meninos iniciaram comportamentos } \\
\text { compatíveis com o diagnóstico de TA. Histórico materno de TA atua } \\
\text { como fator de risco para adolescentes do sexo feminino. Comentários } \\
\text { depreciativos emitidos por meninos foram associados a fator de risco para } 0 \\
\text { desenvolvimento de TA em meninas }\end{array}$ \\
\hline $\begin{array}{l}\text { Canetti et al. } \\
(2008)\end{array}$ & Israel & $\begin{array}{l}33 \text { pacientes com AN e } 33 \text { participantes } \\
\text { (grupo de comparação) }\end{array}$ & Quase-experimental & $\begin{array}{l}\text { Controle materno e cuidado paterno exacerbados foram associados à } \\
\text { gravidade dos sintomas. } 0 \text { cuidado da avó materna foi associado ao } \\
\text { aparecimento de TA. Sugere-se que características dos avós influenciam no } \\
\text { desenvolvimento do TA das netas }\end{array}$ \\
\hline $\begin{array}{l}\text { Franko et al. } \\
(2008)\end{array}$ & Estados Unidos & 2.379 garotas & $\begin{array}{l}\text { Quase-experimental } \\
\text { (longitudinal - } 10 \text { anos) }\end{array}$ & $\begin{array}{l}\text { Refeições familiares mais frequentes sugerem maior coesão familiar e } \\
\text { maior capacidade de desenvolver/aprender estratégias de enfrentamento } \\
\text { saudáveis, o que contribui para reduzir, ao longo dos anos, os níveis de } \\
\text { estresse das filhas, bem como a tendência a desenvolverem sintomas de TA }\end{array}$ \\
\hline $\begin{array}{l}\text { Férnandez-Aranda } \\
\text { et al. (2006) }\end{array}$ & Espanha & $\begin{array}{l}88 \text { pacientes com AN, } 123 \text { pacientes } \\
\text { com BN, } 50 \text { pacientes com TA não } \\
\text { especificado e } 160 \text { participantes } \\
\text { saudáveis (grupo controle) }\end{array}$ & Quase-experimental & $\begin{array}{l}\text { Pessoas com TA relataram padrões alimentares desfavoráveis desde cedo, } \\
\text { com excessiva importância atribuída à comida pela família e pelo indivíduo. } \\
0 \text { consumo de comida especialmente preparada por um familiar para } 0 \\
\text { respondente foi associado ao desenvolvimento de TA }\end{array}$ \\
\hline $\begin{array}{l}\text { Francis et al. } \\
(2005)\end{array}$ & Estados Unidos & 173 díades mãe-filha & $\begin{array}{l}\text { Quase-experimental } \\
\text { (longitudinal - } 6 \text { anos) }\end{array}$ & $\begin{array}{l}\text { Mães que se mostraram mais preocupadas com seu próprio peso e } \\
\text { padrão de alimentação evidenciaram maiores níveis de restrição do } \\
\text { comportamento alimentar das filhas, além de também encorajá-las a } \\
\text { perder peso. Esse encorajamento está associado a maiores níveis de } \\
\text { restrição alimentar nas filhas, que percebem a atitude das mães como } \\
\text { pressão exercida sobre elas }\end{array}$ \\
\hline
\end{tabular}

A maioria dos artigos utilizou instrumentos padronizados (questionários e escalas) para a coleta dos dados. Os resultados evidenciaram a influência que os relacionamentos familiares podem exercer no desenvolvimento e manutenção dos sintomas. Uma parcela expressiva dos artigos sugere que o profissional de saúde deve aprofundar e ampliar o foco na família no planejamento das estratégias interventivas. No que concerne ao relacionamento mãe-filhos, o comportamento alimentar da mãe, suas convicções pessoais acerca do peso e padrões de beleza, bem como seus níveis de satisfação/insatisfação corporal, podem influenciar no surgimento de possíveis comportamentos associados a distúrbios na alimentação, assim como modular a gravidade dos sintomas do TA ${ }^{16-22}$.

\section{DISCUSSÃO}

A estratificação em NE é um procedimento que orienta a elaboração dos graus de recomendações de condutas médicas e reflete o nível de certeza e clareza das publicações, bem como seu poder de modificar e orientar a tomada de decisão final. Em relação às recomendações, o grau de recomendação A está ligado ao NE 1 e sugere forte recomendação na escolha, ou seja, o NE é excelente, o que permite recomendar rotineiramente a conduta. Nesse caso, os benefícios têm peso maior do que o dano, havendo boas evidências para apoiar a recomendação ${ }^{23}$.

Como não foram encontrados artigos com NE 1 nesta revisão sistemática, e a maior parte dos artigos apresenta NE 4, o grau de recomendação deles é C. Logo, são necessários estudos com maiores NE, capazes de fornecer contribuições mais conclusivas e subsídios mais consistentes para a prática profissional na área dos TA. O presente estudo, portanto, encontrou mínimas evidências satisfatórias na análise dos desfechos, o que aponta que os benefícios e riscos não justificam a generalização da recomendação, uma vez que há evidências insuficientes, contra ou a favor.

Por outro lado, deve-se considerar que foram encontrados também alguns estudos com NE 3, os quais recomendam ações que incluem a investigação da transmissão psíquica intergeracional, uma vez que pesquisas com tal temática mostram evidências importantes no desfecho, sugerindo que os benefícios superam os riscos. Assim, há também evidências razoáveis para apoiar a recomendação de estudos futuros, de modo que o profissional de saúde possa incorporá-las à sua prática. Tais resultados orientam o manejo clínico de outros pacientes em condições semelhantes. 
Tabela 2. Caracterização dos artigos com NE 4 segundo primeiro autor/ano de publicação, origem, características dos participantes, delineamento metodológico do estudo e principais resultados

\begin{tabular}{|c|c|c|}
\hline Autoria/Ano & Origem & Participantes \\
\hline $\begin{array}{l}\text { Blissett et al. } \\
\text { (2011) }\end{array}$ & Reino Unido & $\begin{array}{l}23 \text { díades pai-mãe de crianças } \\
\text { entre } 18 \text { meses e } 5 \text { anos }\end{array}$ \\
\hline $\begin{array}{l}\text { Anschutz et al. } \\
\text { (2009) }\end{array}$ & Holanda & $\begin{array}{l}501 \text { crianças de ambos os sexos } \\
\text { entre } 7 \text { e } 10 \text { anos }\end{array}$ \\
\hline $\begin{array}{l}\text { Souza et al. } \\
(2009)\end{array}$ & Brasil & $\begin{array}{l}37 \text { familiares de pessoas com TA, } \\
\text { participantes de } 10 \text { sessões de } \\
\text { terapia de grupo de um serviço } \\
\text { especializado }\end{array}$ \\
\hline $\begin{array}{l}\text { Sherkow et al. } \\
(2009)\end{array}$ & Estados Unidos & 4 díades mãe-filha \\
\hline $\begin{array}{l}\text { Ahrén-Moonga et } \\
\text { al. (2009) }\end{array}$ & Suécia & $\begin{array}{l}14.193 \text { homens e mulheres } \\
\text { nascidos entre } 1915 \text { e } 1929 \\
\text { (primeira geração), } 13.376 \\
\text { mulheres nascidas entre } 1952 \text { e } \\
1989 \text { (terceira geração) - } 55 \text { delas } \\
\text { com TA. Não há informações de } \\
\text { quantos participantes da segunda } \\
\text { geração foram analisados }\end{array}$ \\
\hline
\end{tabular}

$\begin{array}{lll}\text { Jacobi et al. } & \text { Alemanha } & 426 \text { crianças de ambos os } \\ \text { (2008) } & \text { sexos entre } 8 \text { e } 12 \text { anos e seus } \\ & \text { cuidadores primários ( } 91 \% \text { mães) }\end{array}$

Dallos et al. Reino Unido Quatro famílias nas quais um (2008)

Cooley et al. Estados Unidos 91 díades mãe-filha (em idade (2007)

Benninghoven et Alemanha al. (2007)

Hanna et al. Austrália 315 mulheres entre 14 e 28 anos

(2006)

Honey et al. Austrália (2006)

Keery et all. (2005) Estados Unidos

372 garotas que cursavam middle school

24 adolescentes do sexo feminino diagnosticadas com AN, 16 mães e 8 pais

Qualitativo, descritivo, exploratório

Não experimental, descritivo, correlacional

Não experimental, descritivo correlacional

Qualitativo, descritivo, exploratório

Não experimental, descritivo, correlacional

Não experimental, descritivo correlacional

Não experimental, descritivo, correlacional

Qualitativo, descritivo, exploratório

Não experimental, descritivo correlacional

Barnett et al. Reino Unido 0ito mães diagnosticadas com TA (2005)
Qualitativo, descritivo, exploratório
Meninas com alto IMC evidenciaram mais problemas com alimentação e peso e maior insatisfação corporal do que garotos ou crianças mais novas. Filhas de mulheres com sobrepeso restringem mais sua alimentação. Foram sugeridas intervenções preventivas para filhos de mulheres com sobrepeso ou com comportamentos alimentares descontrolados

Foram encontradas relações familiares assentadas em uma base de realidade frágil, padrões transgeracionais de relacionamentos problemáticos, conflitos, discussões e triangulação e relações negativas com 0 alimento. Nas quatro famílias a pessoa com AN apareceu como aquele membro familiar que constela uma experiência corretiva dos próprios pais em relação a experiências negativas vivenciadas em suas infâncias

As mães apresentaram avaliações negativas do corpo de suas filhas e desaprovação de sua figura, o que aumentou significativamente a dificuldade das filhas em tais áreas. Mães que internalizaram mensagens da mídia sobre magreza estariam mais propensas a terem filhas com TA

Em ambos os grupos de pacientes investigados, aspectos do funcionamento familiar foram associados à distorção e insatisfação corporal, detectadas tanto nas mães como nas filhas

Mensagens negativas são importantes fatores que influenciaram no desenvolvimento da sintomatologia do TA, bem como da autoimagem negativa, mais do os que conflitos familiares em si

Irmãos podem influenciar suas irmãs doentes de maneira positiva ou negativa, principalmente no que tange ao conhecimento dos irmãos em relação à doença, suas reações em relação aos sintomas, estilo de vida, encorajamento/ desencorajamento de alguns comportamentos das irmãs e relacionamentos interpessoais

Em famílias em que o pai faz comentários provocativos à filha (em relação ao peso e imagem corporal), é mais provável que o irmão também o faça, sugerindo a transmissão intergeracional do comportamento. Os comentários negativos também influenciam a autoestima, comportamentos alimentares patológicos e sintomas depressivos

Apenas um caso foi apresentado com maior ênfase, e os pesquisadores observaram e analisaram que a mãe encontrou no grupo (para mães com TA) um espaç̧o de reflexão. Ela pôde se conscientizar e reavaliar seus comportamentos alimentares, ressignificando as mensagens negativas que transmitia a seus filhos em relação à alimentação 
Em relação ao conteúdo dos artigos revisados, três deles apontaram padrões de transmissão de comportamentos e sintomas em três gerações: avós, pais e filhos ${ }^{24-26}$. Esses artigos apresentavam NE 4, 3 e 3, respectivamente, e revelaram que a conduta dos avós pode influenciar na gravidade dos sintomas e nos riscos de hospitalização das netas. Todos os artigos restantes abordaram as relações familiares em apenas dois grupos: pais-filhos, mães-filhos, ou entre irmãos. Trata-se de uma limitação importante, na medida em que restringe a apenas duas gerações o alcance dos resultados da maioria dos artigos selecionados.

Dois dos artigos revisados avaliaram a influência dos irmãos na sintomatologia do TA, apresentando NE 4. Os artigos em questão revelam que os irmãos podem atuar como influências negativas quando tecem comentários provocativos a respeito do peso/aparência da irmã acometida, disparando ou intensificando conflitos familiares preexistentes ${ }^{21,27}$. Os irmãos também podem exercer influência positiva, pois muitas vezes são vistos como exemplo a ser seguido pela irmã diagnosticada com AN, uma vez que, na presença deles, elas passavam a perceber melhor a gravidade de seu quadro clínico. Os irmãos adultos auxiliam suas irmãs confrontando seus pensamentos afastados da realidade, expressando suas preocupações e opiniões, bem como encorajando-as a se tratarem ${ }^{27}$.

Um achado importante se refere ao fato de dois artigos, com NE 3 e $4^{21,28}$, terem refutado a hipótese de que comentários negativos ou implicâncias com o peso de adolescentes do sexo feminino por parte das mães estariam associados ao desenvolvimento de TA. Porém, comentários críticos por parte dos pares do sexo masculino se revelaram associados a efeitos negativos nas adolescentes ${ }^{28}$, bem como comentários emitidos por familiares (pais e irmãos) do sexo masculino ${ }^{21}$. Não obstante, é preciso salientar que ambos os artigos em questão apresentam uma limitação importante, pois, em contraste com o que seria indicado em função de seus objetivos, prescindiram de diferentes informantes.

De qualquer forma, um desses artigos mostrou que, nas famílias em que o pai é quem provoca a filha com comentários pejorativos, é muito mais provável que haja um irmão provocador, evidenciando um padrão de transmissão intergeracional do comportamento paterno. O pai e o irmão mais velho, considerados modelos de interação "heterossocial", geram os principais resultados negativos para as garotas suscetíveis ao TA. O artigo que reporta tais achados sugere o desenvolvimento de programas preventivos em relação a esses comportamentos ${ }^{21}$. De acordo com o outro artigo no qual comentários negativos das mães não se revelaram associados ao desenvolvimento de TA, também despontaram como fatores de risco para garotas a exposição às mensagens da mídia, a tentativa da adolescente de se parecer com alguma figura ("celebridade") com alta visibilidade nos meios de comunicação de massa e o histórico materno de TA ${ }^{28}$.
No mesmo sentido, quatro artigos buscaram avaliar os comentários (críticas) de familiares em relação ao peso e/ ou aparência das adolescentes. Os resultados mostram que pessoas, sobretudo mulheres, que são alvo frequente de comentários pejorativos sobre seu peso por parte de suas mães, têm maior probabilidade de desenvolver sintomas de TA e se mostram mais insatisfeitas com sua autoimagem ${ }^{17-19,29}$. Os NE dos artigos em questão foram 4, 3, 3 e 4, respectivamente. Contudo, é preciso salientar que apenas dois deles ${ }^{17,18}$ incluíram entre os participantes díades mãe-filha, o que pode ser apontado como uma limitação.

Apenas três estudos avaliaram a influência de comportamentos e comentários dos pais, e não apenas das mães, nos filhos ${ }^{21,28,30}$, e apresentaram NE 4, 3 e 4, respectivamente. Os resultados foram positivos para influência apenas em um dos artigos, o qual aponta que comentários paternos sobre o peso/aparência da filha são mais prejudiciais do que comentários vindos das mães ${ }^{21}$. Vale reforçar, entretanto, que, como já mencionado, o artigo em questão prescindiu de diferentes informantes.

Outros artigos revelam que mães insatisfeitas com sua autoimagem corporal (por apresentarem sobrepeso, desejo de emagrecer, distorção da imagem do corpo), e/ ou com sintomas na esfera do comportamento alimentar (restrição ou descontrole) têm filhas mais insatisfeitas com seus próprios corpos, o que aumenta as chances de desenvolvimento de $T A^{16,17,20,31}$. Os artigos em questão adotaram delineamento quantitativo, envolveram a aplicação de questionários padronizados e contaram com amplas amostras, o que deve ser apontado como um aspecto positivo. Todos eles apresentam NE 4. Os resultados obtidos, de modo geral, evidenciam que a gravidade da sintomatologia da mãe tem influência direta na gravidade dos sintomas da filha ${ }^{17}$. É possível perceber que a insatisfação da mãe com sua autoimagem pode ter intenso impacto em seus filhos, porém não foram encontrados estudos que buscaram investigar especificamente as percepções das causas atribuídas pelos participantes (mães e filhos) a esse fenômeno de transmissão.

Outros dois artigos, também com NE 4, avaliaram a proposta de grupos preventivos, cujo objetivo era evitar que mães diagnosticadas com TA transmitissem aos filhos suas dificuldades em relação à alimentação e ao corpo. As mães mostraram muitas dificuldades em comprar e preparar os alimentos. Com a participação nos grupos, puderam refletir sobre as condutas que estavam tomando na educação dos filhos e reavaliaram as mensagens ambivalentes que thes transmitiam acerca da alimentação ${ }^{32,33}$. A generalização de tais achados, contudo, deve ser realizada com cautela, pois ambos os estudos contaram com número reduzido de participantes.

Não foram encontrados artigos com propostas preventivas para o fenômeno de transmissão psíquica nas famílias em que não havia uma pessoa diagnosticada com TA. A in- 
satisfação é delegada aos jovens e canalizada por meio de palavras ou atitudes ambivalentes em relação ao alimento. Parece haver uma lacuna no conhecimento científico, que apenas sugere o desenvolvimento de propostas preventivas com famílias de crianças e adolescentes, com intuito de reduzir a frequência desses comportamentos e a possibilidade de desenvolvimento de sintomas.

De acordo com quatro artigos, mães mais preocupadas com seu próprio peso e padrão de alimentação tentam influenciar suas filhas com relação ao peso e alimentação, induzindo-as a adotarem comportamentos de restrição alimentar ${ }^{18,21,30,33}$. Os NE apresentados por tais artigos são 4, 3, 4 e 4, respectivamente. Outros dois artigos, com NE 3, também apontam que a família deve fazer refeições conjuntas frequentes, o que pode ajudar os filhos (crianças e adolescentes) a desenvolverem atitudes e pensamentos mais saudáveis em relação à alimentação, além de ser um importante momento de convivência com a família ${ }^{26,34}$. Um desses artigos também elenca como fatores de risco relacionados ao desenvolvimento de TA ser integrante de família numerosa e conviver com os avós em casa. Este último fator pode estar relacionado a um estilo inadequado de alimentação dos avós direcionado para os netos, em alguns casos permissivos em demasia e, em outros, rígidos e inflexíveis ${ }^{26}$.

Ao avaliar se filhos (bebês) de mulheres diagnosticadas com TA têm dificuldades alimentares no início da vida, considerando os sintomas de TA da mãe, seus níveis de ansiedade e depressão, um artigo reportou que sintomas de TA ativos durante o período de gravidez e histórico prolongado do quadro materno psicopatológico aumentam o risco de problemas alimentares do bebê, podendo ocasionar baixo peso e outros problemas de desenvolvimento ${ }^{35}$. Tal artigo apresenta NE 3 e têm o mérito de se dedicar a um aspecto da temática em pauta que permanece pouco explorado pela literatura.

Dois artigos com delineamento qualitativo, ambos apresentando NE 4, encontraram que as dificuldades alimentares são transmitidas entre as gerações, perseverando um padrão de relação negativa com o alimento. Pais e mães tentaram, em vão, proteger seus filhos de suas dificuldades emocionais e mostraram preocupações em relação a não serem capazes de expressar seus sentimentos ${ }^{33,36}$. Nesse contexto relacional problemático são fomentados segredos familiares, especialmente relacionados aos sintomas alimentares da mãe, que são transmitidos aos filhos ${ }^{33}$.

Em suma, na presente revisão destacou-se o predomínio de artigos quantitativos, com pouca referência aos elementos de transmissão psíquica entre gerações. Os artigos que mais exploraram tais elementos apresentaram abordagem qualitativa justamente por tratarem de um conceito presente na clínica psicanalítica e que se desenvolve, predominantemente, em forma de estudos de casos. Desse modo, em que pese o fato de estudos qualitativos apresentarem apenas NE 4 de acordo com a classificação da AHRQ, é possível propor que talvez eles sejam capazes de fornecer subsídios particularmente profícuos para a discussão sobre o papel do grupo familiar no desenvolvimento da AN, tendo em vista a reconhecida importância dos modelos psicodinâmicos na explicação dos TA. Tal consideração pode ser incluída na agenda de novos estudos, que possam compreender de modo mais pormenorizado a participação da família no contexto do tratamento.

Vale destacar, ainda, que os artigos que constituíram o corpus que deu suporte a esta revisão indicam a importância da influência dos familiares na sintomatologia da AN. Ainda assim, somente um estudo apresentava uma proposta interventiva de prevenção de transmissão de comportamentos alimentares inadequado ${ }^{32}$. Os demais artigos apenas apontaram a necessidade de cuidados preventivos ${ }^{19-21,26,28,29,31,34}$, principalmente nas escolas (coibindo as práticas de bullying, por exemplo) e dentro do grupo familiar, mas a literatura científica encontrada ainda não acompanha tal demanda. Essa situação evidencia uma importante limitação dos artigos revisados no presente estudo, que é justamente não possibilitar a compreensão da transmissão psíquica como um modelo teórico exequível de ser instrumentalizado na terapêutica desses pacientes, a fim de permitir a abordagem dos elementos familiares relacionados à sintomatologia, ao desenvolvimento, tratamento e recuperação das pessoas acometidas pela AN.

\section{CONCLUSÕES}

Nesta revisão foram avaliadas, criticamente, as evidências científicas disponíveis sobre a influência do grupo familiar na sintomatologia da AN. Os resultados permitem destacar que os relacionamentos familiares exercem impacto significativo no desenvolvimento e manutenção dos sintomas de TA. Foram encontradas evidências de que padrões relacionados à alimentação e ao alimento são transmitidos entre as gerações, bem como aspectos não elaborados do funcionamento psíquico materno.

A classificação em NE facilita o entendimento da atual maturidade do conhecimento na área, ou seja, o grau de sua recomendação clínica. Nesta revisão foram encontradas mínimas evidências satisfatórias na análise dos desfechos, o que permite concluir que a relação risco-benefício não justifica a generalização da recomendação. Em que pese a insuficiência de evidências contra ou a favor, e a inexistência de estudos com delineamentos robustos, os achados dos estudos revisados devem ser levados em consideração, com a devida cautela, pelos profissionais da equipe multidisciplinar no momento de definirem estratégias de cuidado em saúde que incluam a família no plano de tratamento.

Essa inclusão de membros da família deve se dar não apenas considerando-os como fonte privilegiada de infor- 
mações sobre a pessoa acometida pelo TA, mas como reconhecimento das implicações familiares no desenvolvimento e manutenção dos sintomas. Acredita-se que essas medidas possam favorecer melhor entendimento do manejo do esquema terapêutico e, consequentemente, estimular a adesão dos pacientes ao tratamento prescrito e à adoção de comportamentos protetores de saúde. Esses conhecimentos podem direcionar os profissionais da equipe multidisciplinar de saúde na tomada de decisões, em consonância com as necessidades de cada etapa do tratamento, considerando as repercussões familiares do TA.

Há necessidade de se desenvolverem novos estudos para que os programas de ação em saúde mental possam utilizar continuamente as melhores evidências disponíveis na realidade da prática clínica diária. Desse modo, será possível estruturar protocolos de condutas de maneira judiciosa. Os profissionais da área de saúde devem estar familiarizados com os NE e com os graus de recomendação das publicações científicas. Nesse contexto, investigar a influência familiar no cenário dos TA, especialmente na AN, favorece a sistematização do conhecimento necessário para subsidiar a prática em saúde mental. Ampliar e qualificar a produção de conhecimento nessa área pode contribuir para a identificação precoce dos sintomas nos serviços de saúde, bem como para o planejamento de possíveis ações de promoção da saúde.

\section{CONTRIBUIÇÕES INDIVIDUAIS}

Élide Dezoti Valdanha - Desenhou o estudo, realizou a coleta e análise dos artigos selecionados, bem como a redação e a formatação do manuscrito.

Fabio Scorsolini-Comin - Colaborou com a análise dos artigos selecionados e participou da revisão do manuscrito.

Rodrigo Sanches Peres - Colaborou com a análise dos artigos selecionados e participou da revisão do manuscrito.

Manoel Antônio dos Santos - Desenhou o estudo, orientou o desenvolvimento da pesquisa e colaborou com a análise dos artigos, redação, formatação e revisão do texto.

\section{CONFLITOS DE INTERESSES}

Declaramos que não existem conflitos de interesse (profissionais, financeiros e benefícios diretos ou indiretos) que possam influenciar os resultados da pesquisa.

Declaramos, ainda, que este estudo foi financiado pela Coordenação de Aperfeiçoamento de Pessoal de Ensino Superior (Capes), que concedeu bolsa de estudos, em nível de mestrado, à primeira autora, sob a orientação do último autor, para realização da pesquisa que originou este artigo.

\section{AGRADECIMENTOS}

Os autores agradecem à Coordenação de Aperfeiçoamento de Pessoal de Ensino Superior (Capes), pela concessão de bolsa de estudos, em nível de mestrado, à primeira autora, sob a orientação do último autor, para a realização do estudo que originou este artigo.

\section{REFERÊNCIAS}

1. Roserberg W, Donald A. Evidence based medicine: an approach to clinical problem-solving. BMJ. 1995;310(6987):1122-6.

2. McGinn TG, Guyatt GH, Wyer PC, Naylor CD, Stiell IG, Richardson WS. The evidence-based medicine working group. Users' guides to the medical literature XXII: how to use articles about clinical decision rules. JAMA. 2000;284:79-84.

3. Galvão CM, Sawada NO, Mendes IA. A busca das melhores evidências. Rev Esc Enferm USP. 2003;37(4):43-50.

4. Hughes RG, editor. Patient safety and quality: an evidence-based handbook for nurses. AHRQ Publication n0 08-0043. Rockville, MD: Agency for Healthcare Research and Quality; 2008.

5. Oliveira EA, Santos MA. Perfil psicológico de pacientes com anorexia e bulimia nervosas: a ótica do psicodiagnóstico. Medicina (Ribeirão Preto). 2006;39(3):353-60.

6. Polivy J, Herman (P. Causes of eating disorders. Ann Rev Psychol. 2002;53(1):187-213.

7. Benghozi P. Malhagem, filiação e afiliação - Psicanálise dos vínculos: casal, família, grupo, instituição e campo social. São Paulo: Vetor; 2010.

8. Eiguer A. Um divã para a família. Porto Alegre: Artes Médicas; 1985.

9. Kaës R. Transmissão da vida psíquica entre gerações. São Paulo: Casa do Psicólogo; 2001.

10. Lisboa AV, Féres-Carneiro T. Quando 0 adoecimento assombra 0 grupo familiar. Pulsional Rev Psicanál. 2005;18(184):40-8.

11. Wagner A. Introdução. In: Wagner A, editor. Como se perpetua a família? A transmissão dos modelos familiares. Porto Alegre: EDIPUCRS; 2004.

12. Almeida MES. Uma proposta sobre a transgeracionalidade: 0 absoluto. Ágora. 2010;13(1):93-108

13. Wagner A, Falcke D. A dinâmica familiar e o fenômeno da transgeracionalidade: definição de conceitos. In: Wagner A, editor. Como se perpetua a família? A transmissão dos modelos familiares. Porto Alegre: EDIPUCRS; 2004

14. Lane RC. Anorexia, masochism, self-mutilation and auto-erotism: the spider mother. Psychoanal Rev. 2002;89(1):101-23.

15. Souza LV, Santos MA. Anorexia e bulimia: conversando com as famílias. São Paulo: Vetor; 2007.

16. Benninghoven D, Tetsch N, Kunzendorf S, Jantscheck $\mathrm{G}$. Body image in patients with eating disorders and their mothers, and the role of family functioning. Compr Psychiatry. 2007:48(2):118-23

17. Cooley E, Toray T, Wang MC, Valdez NN. Maternal effects on daughter's eating pathology and body image. Eat Behav. 2007;9(1):52-61.

18. Francis $L A$, Birch LL. Maternal influences on daughter's restrained eating behavior. Health Psychol. 2005;24(6):548-54

19. Hanna AC, Bond MJ. Relationships between family conflict, perceived maternal verbal messages, and daughters' disturbed eating symptomatology. Appetite. 2006;47(2):20511.

20. Jacobi C, Schmitz G, Agras WS. Interactions between disturbed eating and weight in children and their mothers. J Dev Behav Pediatr. 2008;29(5):360-6.

21. Keery H, Boutelle K, Berg P, Thompson JK. The impact of appearance-related teasing by family members. J Adolesc Health. 2005;37(2):120-7.

22. Souza LV, Santos MA, Scorsolini-Comin F. Percepções da família sobre a anorexia e bulimia nervosa. Víncul0. 2009;1(6):26-38. 
23. Phillips B, Ball C, Sackett D, et al. Oxford Centre for Evidence-based Medicine Levels of Evidence Grades of Recommendation (2001). Disponível em: <http://cebm.jr2.ox.ac.uk/ docs/levels4.html> Acesso em: 7 março 2013.

24. Ahrén-Moonga J, Silverwood R, Klitenberg B, Koupil I. Association of higher parental and grandparental education and higher school grades with risk of hospitalization for eating disorders in females. Am J Epidemiol. 2009;170(5):566-75.

25. Canetti L, Kanyas K, Lerer B, Latzer Y, Bachar E. Anorexia nervosa and parental bonding: the contribution of parent-grandparent relationships to eating disorder psychopathology. J Clin Psychol. 2008;64(6):703-16.

26. Férnandez-Aranda F, Krug I, Granero R, Ramón JM, Badia A, Giménez L, et al. Individual and family eating patterns during childhood and early adolescence: an analysis of associated eating disorder factors. Appetite. 2006;49(2):476-85.

27. Honey A, Clarke S, Halse C, Kohn M, Madden S. The influence of siblings on the experience of anorexia nervosa of adolescent girls. Eur Eat Disord Rev. 2006;14(5):315-22.

28. Field AE, Javaras KM, Aneja P, Kitos N, Camargo CA, Taylor B, et al. Family, peer and media predictors of becoming eating desordered. Arch Pediatr Adolesc Med. 2008;162(6):574-9.

29. Eisenberg ME, Berge JM, Fulkerson JA, Newmark-Sztainer D. Associations between hutful weight-related comments by family and significant other and the development of disorderd eating behaviors in young adults. J Behav Med. 2012;35(5):500-8.
30. Blissett J, Haycraft E. Parental eating disorder symptoms and observations of mealtime interactions with children. J Psychosom Res. 2011;70:368-71.

31. Anschutz DJ, Kanters LJA, Strien TV, Vermulst AA, Engels RCME. Maternal behaviors and restrained eating and body dissatisfaction in young children. Int J Eat Disord. 2009;42(1):54-61.

32. Barnett S, Buckroyd J, Windle K. Eating disorders from parent to child: mother's perceptions of transgenerational effect. Couns Psychother Res. 2005;5(3):203-11.

33. Sherkow SP, Kamens SR, Megyes M, Loewenthal L. A clinical study of the intergenerational transmission of eating disorders from mothers to daughters. Psychoanal Study Child. 2009;64:153-89

34. Franko DL, Thompson D, Barton BA, Affenito SG, Striegel-Moore RH. What mediates the relationship between family meals and adolescent health issues? Health Psychol. 2008;27(2):109-17.

35. Micali N, Simonoff E, Stahl D, Treasure J. Maternal eating disorders and infant feeding difficulties: maternal and child mediators in a longitudinal general population study. J Child Psychol Psychiatry. 2011;52(7):800-7.

36. Dallos R, Denford S. A qualitative exploration of relationship and attachment themes in families with an eating disorder. Clin Child Psychol Psychiatry. 2008;13(2):305-22. 